\title{
An Alternative Explanation for Diffractive Imaging Technique
}

\author{
Xiaodong Tao, Joseph Wall and Yimei Zhu \\ Brookhaven National Laboratory, Upton, NY 11973
}

In recent years, the idea of using iteration algorithms [1,2] to retrieve the exit wave from a coherent diffraction pattern of a small object has regained attention. A series of experimental success has been made in both X-ray and electron diffraction [3]. The most popular explanation for this technique, often quoted as diffractive imaging or oversampling method, is based on the oversampling argument by Sayre [4]. In this paper, we give a more intuitive explanation on how the phase is encoded into the diffraction pattern based on the principle of Ptychography. An alternative algorithm is designed to illustrate this idea. It explains that the "twin image" problem associated with holography shows in a different form for diffractive imaging.

For a crystalline specimen, Ptychography, developed by Hoppe[5], involves modifying the electron beams to generate interferences among the Bragg orders. Hoppe planned to apply this technique to TEM, however, only its application to CBED patterns in STEM was well recognized and systematically studied [6]. Under kinematical assumption, the intensity distribution of a CBED pattern can be approximated as : $\mathrm{I}(\mathrm{K})=|\mathrm{F}(\mathrm{K}) \otimes \mathrm{H}(\mathrm{K})|^{2}$, where $\mathrm{F}(\mathrm{K})$ being the structure factors associated with a series of discrete Bragg reflections, and $\mathrm{H}(\mathrm{K})$ is the aperture function including the beam aberration. Each diffraction disk is the convolution product of a Bragg spot with an aperture function. If the neighboring diffraction disks overlap (interfere) with each other, one or more paths can be built among all of the diffraction disks and the phase information of the structure factor can be determined. This concept is illustrated in Fig.1. If the diffraction disks do not overlap, the phase information of the structure factors is lost in a kinematical approximation.

Figure 2 shows the experimental setup for diffractive imaging -- a parallel and coherent beam illumination on an object. For simplicity, we only consider a small crystalline specimen that can be treated as a small part of an infinite crystal whose phase grating effect is defined as $\mathrm{f}(\mathrm{x})$. The shape of the specimen is defined by a top hat function, $\mathrm{g}(\mathrm{x})$, which has values 1 or 0 . Thus, the exit wave is expressed as $\mathrm{f}(\mathrm{x}) \cdot \mathrm{g}(\mathrm{x})$. Assuming that the Ewald sphere is flat, the diffraction pattern at the far field can also be expressed as the square of a convolution product, $\mathrm{I}(\mathrm{K})=|\mathrm{F}(\mathrm{K}) \otimes \mathrm{G}(\mathrm{K})|^{2}$. For a small crystalline sample, each Bragg peak is broadened through the convolution with $\mathrm{G}(\mathrm{K})$. Ideally, the function $\mathrm{G}(\mathrm{K})$ has a complex shape extending to infinity. For the discrete case and under practical experimental conditions, $\mathrm{G}(\mathrm{K})$ is spatially limited and can be described by a finite number of pixels. If the broadened Bragg peaks do not overlap, or the signal to noise ratio is too low in the overlapped region, the phase information is lost in the diffraction pattern and infinite numbers of solution exist for the iteration algorithm. Similar to the Ptychography, the overlapping of the Bragg peaks encoded the phase into the diffraction pattern, as shown in Fig.3. The diffraction intensity of a non-periodic object doesn't include Bragg peaks, instead it is assumed to be continuous in the reciprocal space. Thus, an infinite hypothetical crystal could be assumed to be $\mathrm{f}(\mathrm{x})$, whose diffraction pattern is discontinuous. Convolution of this discontinuous function with the Fourier transform of the shape function caused extensive interference, which generates a continuous diffraction pattern. Based on this argument, we designed an alternative iteration algorithm. Instead of retrieving the exit wave $\mathrm{f}(\mathrm{x}) \cdot \mathrm{g}(\mathrm{x})$, this algorithm is designed to find $\mathrm{f}(\mathrm{x})$. Numerical simulation using this algorithm is successful, as shown in Fig.4. As a pre-requisite, this algorithm requires $\mathrm{f}(\mathrm{x})$ to be periodic. This is consistent with the oversampling argument.

For two waves interfering with each other, the intensity in the interference region records the phase difference in the form of a cosine operation. Since cosine is an even function, an ambiguity is introduced when the sign of the phase needs to be determined. This is the origin of the twin image problem in holography. According to this explanation, this problem shows in a different form in diffractive imaging. If $\mathrm{G}(\mathrm{K})$, the Fourier transform of the shape function, is real, the interference of 
two Bragg peaks $\mathrm{G}(\mathrm{K}) \otimes \mathrm{F}\left(\mathrm{K}_{1}\right)$ with $\mathrm{G}(\mathrm{K}) \otimes \mathrm{F}\left(\mathrm{K}_{2}\right)$ only records the absolute value of the phase difference between $\mathrm{F}\left(\mathrm{K}_{1}\right)$ and $\mathrm{F}\left(\mathrm{K}_{2}\right)$. So, for a specimen having a center symmetric shape, both the true wave and its complex conjugate are the right answer to the iteration algorithm. A simple extension of this explanation can be applied on the through focal series technique in HRTEM.

\section{References:}

[1] R.Gerchberg and W.Saxton, Optik(Stuggart), 35 (1972), 237-249

[2] J.R.Fienup, Appl. Optics(1982), 21, P2758-2769

[3] J.M.Zuo, I.Vartanyants, M.Gao, R.Zhang, L.A.Nagahara, Science, V300. P1419-P1421

[4] D.Sayre, Some implications of a theorem due to Shannon, Acta Cryst.(1952). 5, P843

[5] W.Hoppe, Acta Cryst.(1969). A25, P495-501

[6] J.M.Rodenburg and R.H.T.Bates, Phil. Trans.R.Soc.Lond.A(1992)339, P521-523

[7] Finance Support from BNL, US DOE LDRD Project BNL\#04-061, is greatly appreciated.

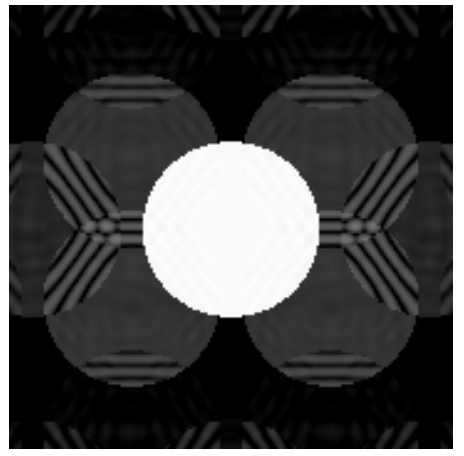

Fig.1. A simulated CBED pattern showing overlapping of the diffraction disks encoded the phase information.
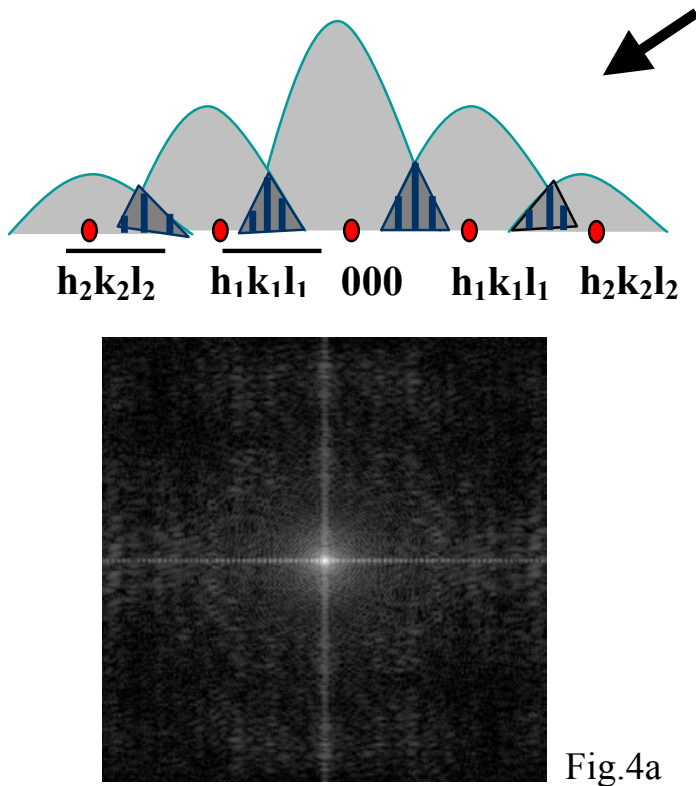

Fig.4a

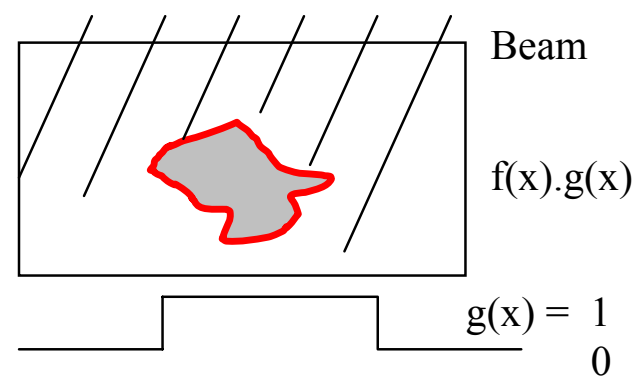

Fig.2. Principle of diffractive imaging : --a parallel wave illuminating a small object. Function $\mathrm{g}(\mathrm{x})$ describes the shape of the object. Exit wave: $f(x) \cdot g(x)$

Fig.3. Overlapping of the broadened Bragg peaks encodes the phase for diffractive imaging. Small solid circles represent Bragg positions

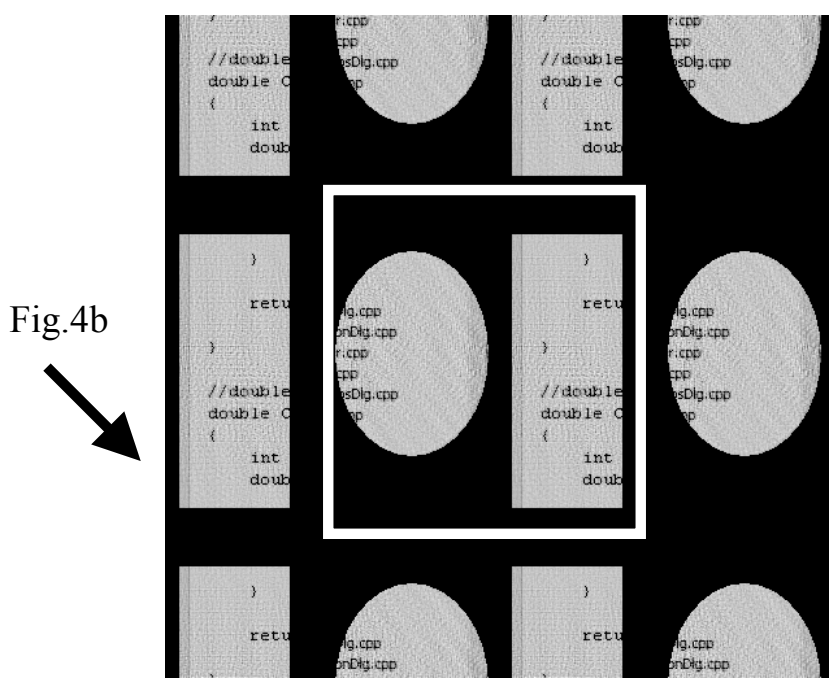

Fig.4 (a) Power spectrum of a testing "non-periodic" object; (b) Numerical reconstruction of the testing object using the power spectrum shown in (a). An alternative algorithm was used. The testing object (not shown here) includes letters which is similar to the contents in the white box. The ellipse and rectangle shown in the white box are used as the shape function. 